\title{
Audiovisual Multimedia Combined with Standard, Verbal, Informed Consent; Enhancing the Satisfaction and Comprehension of Surgical Patients: A Comparative Trial in Patients Undergoing Laparoscopic Cholecystectomies at the HRH Princess Maha Chakri Sirindhorn Medical Center, Thailand
}

\author{
Wuttichai Thanapongsathorn", Chayapa Imchit, Jilapon Phunya \\ HRH Princess Maha Chakri Sirindhorn Medical Center, Department of Surgery, Faculty of Medicine, \\ Srinakharinwirot University, Nakorn-Nayok, Thailand \\ Email: ${ }^{*} w u t t i c h a @$ hotmail.com
}

Received May 26, 2012; revised June 30, 2012; accepted July 12, 2012

\begin{abstract}
Objective: A comparative study of the audiovisual, multimedia, informed consent procedure enhancing the satisfaction and comprehension in laparoscopic cholecystectomy patients within the contexts of the Thai culture. Methods: A prospective, comparative trial was done in patients undergoing laparoscopic cholecystectomies at the HRH Princess Maha Chakri Sirindhorn Medical Center in Thailand. Before the procedure, all patients who met the selection criteria and agreed to participate in the study by signing the proper consent forms were placed into 2 groups. G1 (group 1) was informed with the standard verbal informed consent and G2 (group 2) was informed with audiovisual multimedia in the form of a DVD in collaboration with the standard, verbal, informed consent procedure. The patients were interviewed by a nurse, using a checklist type of questionnaire, as to their satisfaction and comprehension of the procedure on the day of discharge. Results: In the period of June 2010 through December 2010, 60 patients undergoing laparoscopic cholecystectomies and who had met the conditions of research were included in our study. There were 30 patients in each group. The average satisfaction score in G1 was 3.12 out of a total of 5 and in G2 was 4.51 of $5(\mathrm{P}<0.001)$. The percentage of patients that had an understanding of the operative information: 1) Understood $\geq 50 \%$ of information; G1 was at $13.33 \%$ and $\mathrm{G} 2$ was at $70.00 \%$; 2) comprehension of $1 \%-49 \%$ of the information; G1 was at $20.00 \%$ and G2 was at $26.67 \%$; 3 ) No comprehension; G1 was measured at $66.67 \%$ and G2 was at $3.33 \%$. The subgroup included the analysis of 7 items of information (operative indications, operative process, operative results, possible complications, wound care, diet, conditions for returning to work) and these were understood at a higher level in G2 than those in G1 $(\mathrm{P}<0.001)$. Conclusions: In the context of the Thai culture; high technology, audiovisual multimedia combined with the standard, verbal, informed consent process enhanced the satisfaction and the comprehension of patients undergoing a laparoscopic cholecystectomy. Audiovisual multimedia should have the appropriate content, be clear, comprehensive and should include images and the voice of the surgeon who will be performing the operation to help the patient feel confident in the surgeon and in their decision to proceed with the surgery. Providing information in DVD form will benefit patients as they can examine it by themselves in the comfort of their home before the surgery and review the material as many times as may be required to provide a full understanding.
\end{abstract}

Keywords: Audiovisual; Multimedia; Informed Consent; Standard Verbal Informed Consent; Surgical Patient's Satisfaction; Comprehension

\section{Introduction}

Providing patients with the appropriate information prior to any surgery is the standard of care in preparing the body and the mind for the upcoming surgery. In particular, for

${ }^{*}$ Corresponding author. non-emergency patients, information on the procedure and the disease process is provided in an effort to help minimize postoperative complications. Nowadays, the standard is to give a verbal explanation to the patient with explanations of the disease, surgical treatments, and possible alternative treatments as an aid to decrease stress and 
anxiety. This helps to increase patient cooperation and assures the patients' knowledge of their choices.

The surgeon provides the patient with explanations on the following items:

1) Why do you need the surgery?

2) Are there any alternative treatments?

3) Which method will be used in performance of your surgery?

4) What are the anticipated outcomes of the surgery?

5) What are the possible complications that may occur during and after the surgery?

6) What aftercare instructions does the patient need following the surgery?

This process nurtures a mutual understanding between the patient and the health care team in an attempt to minimize patient anxiety and avoid possible legal action against the health care organization in the future. This is particularly relevant when the surgery outcomes are contrary to the patients' expectations. Giving the patient a DVD containing all the pertinent information; images and descriptions of the actual procedure, preoperative preparation and the expected outcomes of the surgery will enhance the cooperation of patients. If the patient is well informed, understands the information in detail, and follows the directions correctly; the patient would exhibit a positive outcome and show satisfaction with the surgical experience. In cases of dissatisfaction with the surgery's outcome, litigation could be avoided or reduced.

Informed consent (IC) is a legal procedure in that a patient voluntarily gives the legal right to the physician to take a medical history, perform a physical examination, perform a medical procedure, make use of specialized instrumentation and laboratory assays in order to diagnose and treat a disease. For example, the physician may ask for informed consent to treat with pharmaceutical substances, surgery, or with other methods that may transgress the patient's personal right to privacy and their health (mental, social, and spiritual) [1-3]. Patients need to receive the appropriate information in order to make an informed decision independently and must sign the informed consent stating their understanding. Then, they have legally given the consent to the physician who will be taking the responsibility and making the decisions on the behalf of the patient. In the case where the patient is unable to decide by her or himself secondary to problems with comprehension, organic or otherwise, the patient's family or close relatives may need to sign the informed consent on the patient's behalf. In emergency cases, in which a surgical procedure must be performed to save the patient's life, the decision is left to the discretion of the attending physician.

Signing a statement of informed consent is an important step in which the patient must receive accurate information and information that is easy to understand to be able to make the right choices and share the responsibility for the events that may occur during or after the surgery, as stated in the patient's rights declaration. In standard practice, the surgeon gives the patient clear and concise information before the surgery. The information is to be given orally with an opportunity for the patient to ask questions. But in the actual practice and in following with the Thai culture, the patient often feels a compulsion not to ask questions, even though the physician may not have explained the matter clearly or with sufficient details. This is because the patient has confidence that the surgeon will perform the surgery in the best way possible, so questions or further information are deemed unnecessary. Also, in the Thai culture, it is considered rude to question a person of authority, such as a physician. In many incidences with persons of authority, Thai people will always smile nod and state agreement even if they do not fully understand what is being told to them. They do this so as not to insult the person of authority as much as not to "lose face" (degrade themselves) by admitting they do not understand what is being told to them. As Thai society changes in accordance with world trends, patient participation in the health care process is steadily increasing. The speed at which the patient receives medical information from websites, internet and from the many different digital social medias, together with the increasing variety of surgical procedures available and the use of a myriad of sophisticated technology opens an opportunity for the patient to research and select their treatment method, choose a hospital, chose a surgeon, and at the same time receive claims for patient's rights. The standard process of delivering information orally may now be inadequate, inappropriate, inefficient or ineffective in providing the patient with information that is easy to understand. Then, it becomes imperative to use modern and diverse technologies such as DVD (Digital Versatile Disc or Digital Video Disc) which includes images and sound. However, the content does not differ from the standard, verbal, informed consent. This allows the surgical patient to watch, listen, and read as many times as necessary before making an informed decision to proceed with the surgery.

However, there is no study, in the context of the Thai culture that addresses the use of audiovisual multimedia for the purpose of obtaining informed surgical consent.

\section{Objectives}

1) Compare the patients' satisfaction between the standard, verbal, informed consent group and the audiovisual multimedia combined with a standard, verbal, informed consent group.

2) Compare the patients' understanding between the standard, verbal, informed consent group and the audiovisual multimedia combined with a standard, verbal, informed consent group. 


\section{Method}

A total of 60 patients that had undergone a non-emergent, laparoscopic cholecystectomy at the HRH Princess Maha Chakri Sirindhorn Medical Center, Faculty of Medicine, Srinakharinwirot University, were assigned to one of 2 groups using the number on the operative schedule: Group 1 (the odd numbered patients on the schedule) consisted of 30 patients who had received the explanation by the standard, verbal method. Group 2 (the even numbered patients on the schedule) consisted of 30 patients who had received the explanation by the standard, verbal method in conjunction with the use of audiovisual multimedia relating to the surgery. The Group 2 patients have had to watch the DVD at least once with the nurse assigned to give this instruction after the surgeon had set the schedule for the surgery. All instruction done in both consent processes were presented in the patients' native language, Thai. The DVD describes the laparoscopic cholecystectomy procedure and is 6.5 minutes in duration and offers an explanation of the specific informed consent forms for this surgery.

The satisfaction level will be scored in five-level Likertscale.

1) Very dissatisfied;

2) Dissatisfied;

3) Neither;

4) Satisfied;

5) Very satisfied.

A Likert scale is a psychometric scale commonly involved in research that employs questionnaires. It is the most widely used approach to scaling responses in survey research.

The patient's level of understanding will be categorized by the nurse using 7 questions:

1) Reasons for having the surgery;

2) Surgical method planned;

3) Expected surgical outcomes;

4) Possible complications during the surgery and postoperatively;

5) Follow up care and prescribed post-operative care;

6) Food that can be taken after the surgery;

7) Returning to work after the surgery.

The result were scored as: 1) Understands $\geq 50 \%$ of the information; 2) Understands $1 \%-49 \%$ of the information, and 3) Not understanding the information at all. The data will be statistical analyzed by t-test with SPSS version 15 . $\mathrm{P}$ value $<0.01$ shows statistical significant.

\section{Results}

In the period of June-December 2010, 60 patients satisfying the conditions of the research were recruited. They were informed of the project and signed a consent form to participate in our study as declared by the ethics committee on 30 June, 2009. Each of the patients in Group 2 viewed the video at least once with the nurse offering explanations as necessary. They were then given a copy of the video to take home to view as needed. We have no data as to the number of times they may have viewed the video on their own. The results are shown in 5 tables:

1) Analysis of sex, age and educational level (Table 1);

2) Analysis of the overall level of satisfaction (Table 2);

3) Analysis of the overall level of comprehension (Table 3);

4) Analysis of the comprehension level, in subgroup data as pertaining to the 7 items asked (Table 4);

5) Analysis of the comprehension level, in subgroup data pertaining to the patient's level of education (Table 5).

\section{Summary}

From the results, we found no statistically significant differences among the two groups in comparisons of sex, age or educational level. The satisfaction level of patients in the group that received the audiovisual information from

Table 1. Sex, age and education level.

\begin{tabular}{|c|c|c|c|}
\hline & & $\begin{array}{c}\text { Group } 1 \\
\text { (did not view the } \\
\text { DVD) }\end{array}$ & $\begin{array}{c}\text { Group } 2 \\
\text { (viewed the DVD) }\end{array}$ \\
\hline Sex & Male:Female & $13: 17$ & $12: 18$ \\
\hline \multirow{4}{*}{ Age } & $<20 y$ & 0 & 0 \\
\hline & $20-39 y$ & 4 & 7 \\
\hline & $40-59 y$ & 14 & 13 \\
\hline & $\geq 60 y$ & 12 & 10 \\
\hline \multirow{3}{*}{ Education } & $\leq$ Primary school & 20 & 13 \\
\hline & Secondary school & 4 & 10 \\
\hline & $\geq$ Bachelor degree & 6 & 7 \\
\hline
\end{tabular}

Table 2. Overall level of satisfaction (likert-scale, scores 1 5).

\begin{tabular}{cc}
\hline Group 1 (did not watch the DVD) & Group 2 (watched the DVD) \\
\hline 3.12 from 5 & 4.51 from 5 \\
\hline & $\mathrm{P}<0.001$
\end{tabular}

Table 3. Overall comprehension level.

\begin{tabular}{lcc}
\hline Comprehension level & $\begin{array}{c}\text { Group 1 } \\
\text { (did not watch the DVD) }\end{array}$ & $\begin{array}{c}\text { Group 2 } \\
\text { (watched the DVD) }\end{array}$ \\
\hline Understands $\geq 50 \%$ & $4(13.33 \%)$ & $21(70.00 \%)$ \\
Understands $1 \%-49 \%$ & $6(20.00 \%)$ & $8(26.67 \%)$ \\
Does not understand & $20(66.67 \%)$ & $1(3.33 \%)$ \\
\hline
\end{tabular}


Table 4. Comprehension level, subgroup data pertaining to 7 items.

\begin{tabular}{lccccccccccccccccc}
\hline & \multicolumn{1}{c}{ Group 1 (did not watch the DVD) } & \multicolumn{1}{c}{ Group 2 (watched the DVD) } \\
\cline { 2 - 5 } & 1 & 2 & 3 & 4 & 5 & 6 & 7 & Average $\%$ & 1 & 2 & 3 & 4 & 5 & 6 & 7 & Average \% \\
\hline Understands $\geq 50 \%$ & 0 & 0 & 13 & 0 & 11 & 1 & 0 & 11.90 & 3 & 28 & 28 & 3 & 28 & 28 & 28 & 69.53 \\
Understands $1 \%-49 \%$ & 2 & 17 & 10 & 1 & 5 & 5 & 1 & 19.52 & 26 & 1 & 1 & 26 & 1 & 1 & 1 & 27.14 \\
Does not understand & 28 & 13 & 7 & 29 & 14 & 24 & 29 & 68.58 & 1 & 1 & 1 & 1 & 1 & 1 & 1 & 3.33 \\
\hline
\end{tabular}

The 7 items are (1) Reasons for have the surgery; (2) Surgical method; (3) Expected surgical outcomes; (4) Possible complications during the surgery and post operatively; (5) Follow up care and prescribed post operative care; (6) Food that can be taken after the surgery; (7) Return to work after the surgery.

Table 5. Comprehension level, subgroup data pertaining to education level.

\begin{tabular}{|c|c|c|c|c|c|c|c|c|}
\hline \multicolumn{5}{|c|}{$\begin{array}{c}\text { Group 1 } \\
\text { (did not watch the DVD) }\end{array}$} & \multicolumn{4}{|c|}{$\begin{array}{c}\text { Group } 2 \\
\text { (watched the DVD) }\end{array}$} \\
\hline $\begin{array}{l}\text { Comprehension } \\
\text { level }\end{array}$ & $\begin{array}{c}\leq \text { Primary } \\
\text { school }\end{array}$ & $\begin{array}{l}\text { Secondary } \\
\text { school }\end{array}$ & $\begin{array}{c}\geq \text { Bachelor } \\
\text { degree }\end{array}$ & total & $\begin{array}{c}\leq \text { Primary } \\
\text { school }\end{array}$ & $\begin{array}{l}\text { Secondary } \\
\text { school }\end{array}$ & $\begin{array}{l}\geq \text { Bachelor } \\
\text { degree }\end{array}$ & total \\
\hline $\begin{array}{l}\text { Understands } \\
\quad \geq 50 \%\end{array}$ & 1 & 0 & 3 & 4 & 7 & 8 & 6 & 21 \\
\hline $\begin{array}{l}\text { Understands } \\
1 \%-49 \%\end{array}$ & 3 & 2 & 1 & 6 & 6 & 1 & 1 & 8 \\
\hline $\begin{array}{l}\text { Does not } \\
\text { understand }\end{array}$ & 16 & 2 & 2 & 20 & 0 & 1 & 0 & 1 \\
\hline total & 20 & 4 & 6 & 30 & 13 & 10 & 7 & 30 \\
\hline
\end{tabular}

the DVD in conjunction with the standard verbal, informed consent was significantly higher than that of the group receiving the standard verbal, informed consent alone $(\mathrm{P}<0.001)$. When analyzing their comprehension; 1) Understands $\geq 50 \%$ of information; G1 was at $13.33 \%$ and $\mathrm{G} 2$ was at $70.00 \%$; 2) Understands $1 \%-49 \%$ of information; G1 was at $20.00 \%$ and $\mathrm{G} 2$ was at $26.67 \%$; 3 ) Not understanding the information; G1 was at $66.67 \%$ and G2 was at 3.33\%. When analyzing the data subgroup, separating each of the 7 factors, we found that G2 had a significantly higher level of understanding $(\mathrm{P}<0.001)$.

\section{Discussion}

From the study, two-thirds of the patients who received the standard consent procedure (Group 1) had no understanding of the "operative information". This data confirms our findings that in following with the Thai culture, the patient often feels a compulsion not to ask questions, even though the physician may not have explained the matter clearly or with sufficient details. This is because the patient is confident that the surgeon will perform the surgery in the best way possible, so questions or further information are deemed unnecessary. Also, in the Thai culture, it is considered rude to question a person of authority, such as a physician. We found that the informed consent procedure needs to be improved to include information relevant to a particular surgery. It must be appropriate with the patient's capabilities and their level of education. The patient needs to understand the favorable and unfavorable outcomes before deciding to proceed with or to defer the surgery. Our results suggest that the use of audiovisual multimedia provides more complete information and this helps the patient to have a clearer understanding of the data that the surgeon wants to communicate. It is a more efficient method when compared with the traditional method. Those who watched the DVD (Group 2) exhibited a higher comprehension level in all levels of educational experience. Potential studies show that explanations, when provided by viewing the DVD, are beneficial to patients regardless of their educational level. According to the characteristics of patients in sex, age, and level of education, no profound differences were found between the two groups. There was a statistically significant higher level of satisfaction and understanding in Group 2 when compared to the group using the traditional method alone. Besides, the level of satisfaction in the group of patients that received audiovisual materials was relatively high (4.64 from 5 points) as was the level of comprehension (4.56 from 5 points). The results correlate with research performed in other countries.

Some previous studies revealed that providing the patient with audiovisual information is effective in giving the patient knowledge and understanding before signing the statement of informed consent [4,5] in many different kinds of surgeries such as; laparoscopic cholecystectomies [6,7], gastric banding, knee arthroscopies, and central venous catheterizations. These studies found that the use of multimedia help the patient to gain a better understanding of the disease process and the surgical proce- 
dure, without experiencing any more anxiety that those not receiving the information materials [8-10].

We presented a DVD, containing images and sounds to provide the patient with a full description of the procedure utilizing drawings as a simpler way of communicating. It includes images of surgical patients that have previously received similar treatments. It is a way to build patient confidence and to help them understand each step of the surgical process up to its completion. It is possible that this procedure, as in all procedures, can have unexpected outcomes. As the patient, after seeing and realizing what the surgery entails, they may become anxious, even fearful, and may choose not to proceed with the surgery. However, it is the patient's right to make an informed decision and accept the surgical outcomes in agreement in conjunction with the patient's rights to be involved in their own health care.

Informed consent does not only mean signing a form for performance of the surgery, it is also a procedure by which the surgeon communicates with the patient to build an understanding. This allows the patient an opportunity to, confidently and voluntarily, accept the surgeon of choice for performing the procedure. The patient acknowledges and understands; the reasons for the surgery, the surgical method, the likely outcomes, all possible complications that may occur, and the self-care required after the surgery. The patient then voluntarily signs the consent form.

The process of informed consent from the patient's prospective:

1) The patient acknowledges and understands the information provided by the surgeon;

2) The patient voluntarily decides to accept or refuses having the surgery performed;

3) The patient signs the consent form as evidence.

The process of informed consent from the surgeon prospective:

1) The surgeon explains the surgery procedure to the patient, who acknowledges and understands the information provided;

2) The surgeon lets the patient decide whether or not the surgery should be performed based on the data provided;

3) The surgeon is witness in the signing of the informed consent forms and keeps the documents as evidence.

\section{Recommendations}

1) The contents of the presentation in the DVD shown to patients should be objectively introduced and explained. Where this DVD should include the appropriate information for the patient to decide whether to proceed with the surgery. It should include images and the voice of the surgeon who will be performing the surgery to help the patient feel confident with their choice. Images of the procedure should be included, but it should not show images of an actual operation that may be too graphic, e.g. showing a scalpel making an incision or blood. It should contain only positive images that will help the patient in their understanding of the surgery. Possibly, it could show a patient who has already had a successful, positive outcome. Let that patient explain the procedure instead of the physician and include expressions of the feelings experienced and reservations before and after the surgery.

2) In assessing the level of the patient's understanding, it should be monitored before and after watching the DVD in order to assure the effectiveness of the information provided in the DVD and, whether or not, the patient was able to obtain a better understanding. This is secondary to the fact that the level of understanding will differ among patients. Some patients will already have obtained previous data or knowledge while others will have no prior knowledge at all.

3) Keeping in mind, the types of patients and their learning potential, particularly, with their differences in educational levels and learning abilities, there is a possibility that the reception of the information in the DVD will be inconsistent. In the Thai context of eastern cultures, the patient tends to sign the informed consent solely on their trust in the professional. They believe that the surgeon will perform the operation in the best possible way and with the best of motives.

4) The use of audiovisual multimedia such as DVDs should be promoted as a method to deliver complex information to the patients, especially in those who possess a lower level of education. This can be attributed to the fact that this group may have limited abilities in the comprehension and reading of text materials.

5) Before the surgery, the patient self-studying the DVD at home may be a possible issue that should be discussed. It is unclear whether it has a positive or negative effect. Since the patient has the possibility to view the DVD more than once, he or she will be able to fully comprehend the material. However, the stress and anxiety that could develop in some patients may escalate to the point of their refusal of a surgery that may actually be the required treatment.

6) The DVD should be produced specifically for the specific surgical procedure as there are several techniques for the same operative condition in the case of unforeseen complications. Also, it could affect the patient as their status after the surgery may differ, e.g. wound healing status, the organs at risk during or after surgery, complications, etc. This will be the future trend of surgical care known as a "patient centered approach". At the same time, the patient's risk and safety in their surgical management becomes a more personal experience. 
7) Informing the patient of the incidence of surgical complications with the updated statistical data, will help make the patient understand and cooperate in the treatment process. This will help lower the occurrence of possible legal actions post-operatively.

\section{Author's Declaration of Open Access}

This article is distributed under the terms of the Creative Commons' Attribution Noncommercial License which permits any noncommercial use, distribution and reproduction in any medium, provided that the original author and his or her sources are accredited.

\section{REFERENCES}

[1] W. K. Leclercq, B. J. Keulers, M. R. Scheltinga, P. H. Spauwen and G. J. van der Wilt, "A Review of Surgical Informed Consent: Past, Present, and Future. A Quest to Help Patients Make Better Decisions," World Journal of Surgery, Vol. 34, No. 7, 2010, pp. 1406-1415. doi:10.1007/s00268-010-0542-0

[2] Y. Schenker, A. Fernandez, R. Sudore and D. Schillinger, "Interventions to Improve Patient Comprehension in Informed Consent for Medical and Surgical Procedures: A Systematic Review," Medical Decision Making, Vol. 31, No. 1, 2011, pp. 151-173.

[3] J. J. Mulsow, T. M. Feeley and S. Tierney, "Beyond Consent-Improving Understanding in Surgical Patients," The American Journal of Surgery, Vol. 203, No. 1, 2012, pp. 112-120. doi:10.1016/j.amjsurg.2010.12.010

[4] C. Hutchison, C. Cowan, T. McMahon and J. Paul, "A Randomized Controlled Study of an Audiovisual Patient Information Intervention on Informed Consent and Recruitment to Cancer Clinical Trials," British Journal of
Cancer, Vol. 97, No. 6, 2007, pp. 705-711.

[5] D. A. Wirshing, M. J. Sergi and J. Mintz, "A Videotape Intervention to Enhance the Informed Consent Process for Medical and Psychiatric Treatment Research," The American Journal of Psychiatry, Vol. 162, No. 1, 2005, pp. 186-188.

doi:10.1176/appi.ajp.162.1.186

[6] E. Bollschweiler, J. Apitzsch, R. Obliers, A. Koerfer, S. P. Mönig, R. Metzger and A. H. Hölscher, "Improving Informed Consent of Surgical Patients Using a Multimedia-Based Program? Results of a Prospective Randomized Multicenter Study of Patients before Cholecystectomy," Annals of Surgery, Vol. 248, No. 2, 2008, pp. 205-211. doi:10.1097/SLA.0b013e318180a3a7

[7] D. Wilhelm, S. Gillen, H. Wirnhier, M. Kranzfelder, A. Schneider, A. Schmidt, H. Friess and H. Feussner, "Extended Preoperative Patient Education Using a Multimedia DVD-Impact on Patients Receiving a Laparoscopic Cholecystectomy: A Randomized Controlled Trial," Langenbeck's Archives Surgery, Vol. 394, No. 2, 2009, pp. 227-233.

doi:10.1007/s00423-008-0460-x

[8] C. Eggers, R. Obliers, A. Koerfer, W. Thomas, K. Koehle, A. H. Hoelscher and E. Bollschweiler, "A Multimedia Tool for the Informed Consent of Patients Prior to Gastric Banding," Obesity, Vol. 15, No. 11, 2007, pp. 2866-2873. doi:10.1038/oby.2007.340

[9] M. J. Rossi, D. Guttmann, M. J. MacLennan and J. H. Lubowitz, "Video Informed Consent Improves Knee Arthroscopy Patient Comprehension," Arthroscopy, Vol. 2, No. 6, 2005, pp. 739-743. doi:10.1016/j.arthro.2005.02.015

[10] J. Lee and H. Yi, "The Effect of Video-Assisted Informed Consent for Central Venous Catheterization in the Emergency Department," Annuals of Emergency Medicine, Vol. 51, No. 4, 2008, p. 481. doi:10.1016/j.annemergmed.2008.01.302 